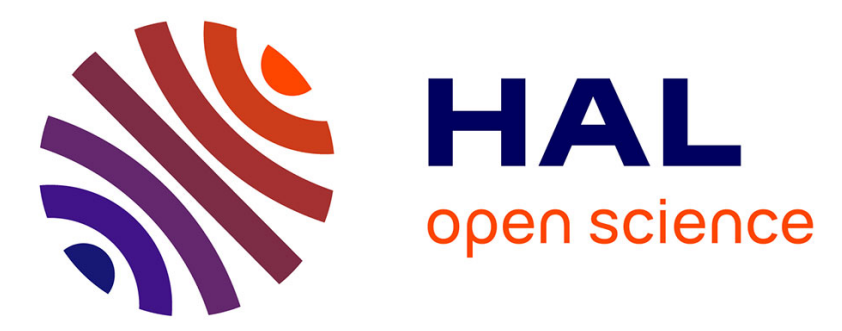

\title{
Informed Source Separation: Underdetermined Source Signal Recovery from an Instantaneous Stereo Mixture
}

\author{
Stanislaw Gorlow, Sylvain Marchand
}

\section{To cite this version:}

Stanislaw Gorlow, Sylvain Marchand. Informed Source Separation: Underdetermined Source Signal Recovery from an Instantaneous Stereo Mixture. 2011 IEEE Workshop on Applications of Signal Processing to Audio and Acoustics (WASPAA 2011), Oct 2011, New Paltz, United States. pp.309312, 10.1109/ASPAA.2011.6082312 . hal-00646276

\section{HAL Id: hal-00646276 https://hal.science/hal-00646276}

Submitted on 22 Apr 2013

HAL is a multi-disciplinary open access archive for the deposit and dissemination of scientific research documents, whether they are published or not. The documents may come from teaching and research institutions in France or abroad, or from public or private research centers.
L'archive ouverte pluridisciplinaire HAL, est destinée au dépôt et à la diffusion de documents scientifiques de niveau recherche, publiés ou non, émanant des établissements d'enseignement et de recherche français ou étrangers, des laboratoires publics ou privés. 


\title{
INFORMED SOURCE SEPARATION: UNDERDETERMINED SOURCE SIGNAL RECOVERY FROM AN INSTANTANEOUS STEREO MIXTURE
}

\author{
Stanislaw Gorlow and Sylvain Marchand
}

\author{
LaBRI CNRS \\ University of Bordeaux 1, 33405 Talence, France \\ firstname.lastnamedlabri.fr
}

\begin{abstract}
The present paper exposes a new technique that aims at solving an ill-posed source separation problem encountered in stereo mixtures. The proposed method is realized in an encoder-decoder framework: On the encoder side, a set of spectral envelopes is extracted from the original tracks, which are known. These envelopes are passed on to the decoder in attachment to the stereo mixture, whereas the frequency resolution of the former is adapted to the critical bands, and their magnitude is logarithmically quantized. On the decoder side, the mixture signal is decomposed by time-frequency selective iterative spatial filtering guided by a source activity index, which is derived from the spectral envelope values. A comparison with a similar algorithm reveals that the novel approach yields a higher perceptual audio quality at a much lower data rate.
\end{abstract}

Index Terms - Inverse problem, informed source separation, stereo, spatial filtering, psychoacoustics

\section{INTRODUCTION}

Blind source separation (BSS) has now been studied for decades and there exists a plethora of literature with just as many different solutions; see [1]. What is common among all BSS algorithms, is the fact that the inverse system, or the "separator", is denied direct access to the sources, forcing it to make additional assumptions, such as statistical behavior (independence, distribution, stationarity, etc.) of the solution. The latter is then found as an optimum with respect to some error measure based on the statistics of the observed data; whether the separation problem is over- or underdetermined. However, when applied to audio, most BSS techniques yield a rather low to intermediate perceptual quality, which disqualifies them from more sophisticated applications like active listening [2] or musical recomposition in general. BSS techniques for speech [3], on the other hand, make assumptions which are generally too restrictive for music. Continuing interest in high-quality solutions for speech and music led consequentially to the emergence of a more recent branch of so-called "informed" source separation (ISS) algorithms, e.g. $[4,5]$, which on the contrary do have access to the sources. Their goal is to find the best possible solution under less stringent assumptions with a minimum of additional information, which is a challenging task. According to the authors, [4] is the state of the art in the context of underdetermined instantaneous linear stereo mixtures. Its basic idea consists in informing the separator, which de facto is a matrix inversion operator, about locally active sound sources, restricting the number of the latter to a maximum of two at the same time. A sparse signal representation is further used to improve the separation performance.

An inherent problem of the above method, however, is that of so-called "spectral holes". These are lacking signal components that occur when more than two simultaneously emitting sound sources contribute to the same frequency content, since all sources apart from the two most predominant are neglected by the separator. The observed effect can be described as a kind of frequency play very similar to the "watery effect" known from music synthesizers, with the difference that here it is the anti-sounds that are produced, which are highly undesired due to their annoying qualities. This effect will further be referred to as the "arid effect". The principal goal of the technique elaborated in this paper is hence to recover "nonperforated" replica of all active sources, which in the broader sense means to drop the assumption of at most two locally predominant sources.

The organization of the paper is as follows: The underlying data model and the problem to be solved is formulated in Section 2. Section 3 gives an in-depth overview of the proposed technique, emphasizing on the most essential processing steps. The simulation results are presented in Section 4. Section 5 concludes the paper by exposing certain shortcomings of the algorithm, leaving room for further discussion.

\section{DATA MODEL AND PROBLEM FORMULATION}

\subsection{Data model}

In the considered scenario, $d$ source signals $\left\{s_{i}(n)\right\}_{i=1}^{d}$ are mixed into a stereo signal $\left\{x_{1}(n), x_{2}(n)\right\}$ through a linear time-invariant (LTI) and memoryless system given by the mixing matrix $\mathbf{A}=$

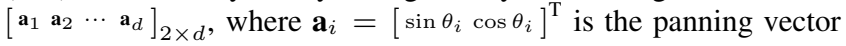
belonging to the $i^{\text {th }}$ source $s_{i}$. Each source is arbitrarily positioned at the unique azimuth $\theta_{i}$ between $0^{\circ}$ (fully right) and $90^{\circ}$ (fully left) in the stereo sound field. Owing to $\left\|\mathbf{a}_{i}\right\|_{2}^{2}=1 \forall \theta_{i}$, the source power level is kept constant over the two channels. By rewriting the set of source signals in vector form, $\mathbf{s}(n)=\left[\begin{array}{llll}s_{1}(n) & s_{2}(n) & \cdots & s_{d}(n)\end{array}\right]^{\mathrm{T}}$, and by doing the same for the stereo signal respectively, $\mathbf{x}(n)=$ $\left[x_{1}(n) x_{2}(n)\right]^{\mathrm{T}}$, the observed system output can be expressed as a plain matrix multiplication,

$$
\mathbf{x}(n)=\mathbf{A s}(n)
$$

\subsection{Problem formulation}

The basic idea is to have an operator or rather an algorithm $\mathcal{A}$ which transforms the mixture signal $\mathbf{x}(n)$ into an approximation $\hat{s}_{i}(n)$ of 
the source signal $s_{i}(n)$ under perceptually motivated constraints $\mathbf{c}\left(\hat{s}_{i}\right)$, while resorting to the knowledge of the mixing system $\mathbf{A}$. Hence, by postulating that perceptually relevant signal parameters $\mathbf{p}_{i}(n)$ be preserved in $\hat{s}_{i}(n)$,

$$
\mathcal{P}: s_{i}(n) \mapsto \mathbf{p}_{i}(n),
$$

where $\mathcal{P}$ is a short-time feature extractor, the problem to be solved can be formulated as follows: Find

$$
\mathcal{A}: \mathbf{x}(n) \times \mathbf{p}_{i}(n) \times \mathbf{A} \mapsto \hat{s}_{i}(n)
$$

subject to

$$
\mathbf{c}\left(\hat{s}_{i}\right)=\hat{\mathbf{p}}_{i}(n)-\mathbf{p}_{i}(n)=\mathbf{0} .
$$

\section{UNDERDETERMINED SOURCE SIGNAL RECOVERY}

\subsection{System overview}

\subsubsection{Encoder}

Within the proposed framework, the sought-after signal parameters represent the sound pressure level (SPL), to which the loudness of a sound, and hence its perceptual significance, is psychologically correlated [6]. The calculation of SPL values is carried out based on a time-frequency representation (TFR) to provide a better disjoint orthogonality [7] of audio signals in comparison to the waveform domain. The processing steps are as follows.

The $i^{\text {th }}$ source signal $s_{i}(n)$ is blockwise time-frequency (TF) analyzed by means of the short-time Fourier transform (STFT) as given by

$$
S_{i}(m, k)=\sum_{n=0}^{L-1} s_{i}(m+n) w(n) e^{-\imath 2 \pi / N k n},
$$

$0 \leqslant k \leqslant N-1$, where $m$ is the block index, $L$ is the length of the window $w(n), \imath$ is the imaginary unit, and $N$ is the transform length $(L \leqslant N)$. The energy spectra $\left\{E_{i}(m, k)\right\}_{i=1}^{d}$ are calculated next from the complex modulus of (5) according to

$$
E_{i}(m, k)=\left|S_{i}(m, k)\right|^{2} .
$$

The latter are further expressed in the form of the energy matrix

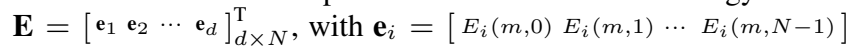
representing the spectral envelope of the $i^{\text {th }}$ source signal in the $m^{\text {th }}$ signal segment. By taking the logarithm, the SPL matrix $\mathbf{L}_{d \times N}$ is put into relation to the energy matrix,

$$
l_{i, n}=10 \log _{10} e_{i, n},
$$

and is subsequently communicated to the decoder together with the $m^{\text {th }}$ local mixture $\mathbf{X}_{2 \times L}$. Depending on $N$, the size of $\mathbf{L}$ can yet become very large, and the amount of additional information may cause a too high data rate. What is more fatal is that imperceptive information hiding may become infeasible even for a high-capacity watermarking technique. The SPL values are therefore "irrelevancy reduced" (see 3.2), as indicated in Fig. 1. Let it be mentioned that the aspect of watermarking is of little relevance for the pursued goal and is therefore excluded from further consideration in the present work.

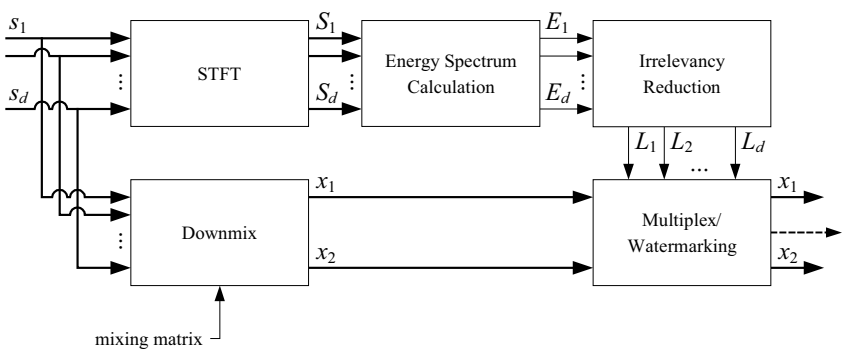

Figure 1: The USSR encoder. $d$ source signals $\left\{s_{i}\right\}$ are transmitted in the downmix $\left\{x_{1}, x_{2}\right\}$ with model parameters $\left\{L_{i}\right\}$ (dashed).

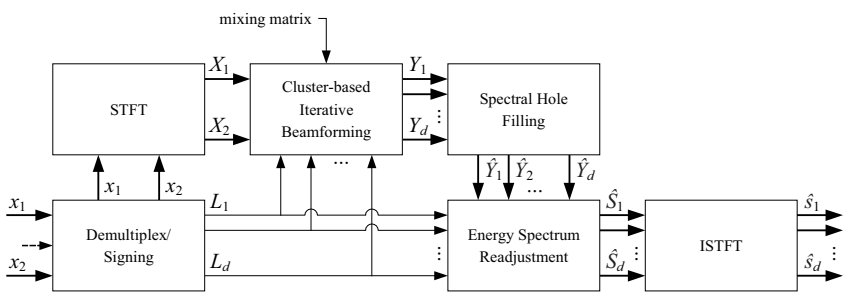

Figure 2: The USSR decoder. Replica of $d$ source signals $\left\{\hat{s}_{i}\right\}$ are recovered from the downmix $\left\{x_{1}, x_{2}\right\}$ by the use of supplementary information $\left\{L_{i}\right\}$ (dashed).

\subsubsection{Decoder}

After splitting the incoming encoder signal back into raw data, $\mathbf{X}$, and parameters, $\mathbf{L}$, the mixture $\mathbf{X}$ is channelwise TF transformed to obtain the TFR matrix $\mathbf{X}^{\emptyset}=\left[\begin{array}{ll}\mathbf{x}_{1}^{\emptyset} & \mathbf{x}_{2}^{\emptyset}\end{array}\right]_{2 \times N}^{\mathrm{T}} . \mathbf{X}^{\emptyset}$ is then iteratively processed by the source separation algorithm, which is the core of the proposed technique (see 3.3). So-obtained source signal spectra $\mathbf{Y}_{d \times N}$ are now fed into the post-processing block to coat residual holes (see 3.4). To guarantee that the post-processed spectra $\hat{\mathbf{Y}}_{d \times N}$ conform to the loudness constraint, the magnitudes of the former are readjusted according to the signaled SPL values $\mathbf{L}$. Finally, the readjusted signal spectra are transformed back to time domain by means of the inverse STFT (ISTFT). A functional block diagram of the decoder is illustrated in Fig. 2.

\subsection{Side information minimization}

A significant reduction of side information can be achieved in two ways: by reducing the frequency resolution of the energy spectra $\mathbf{E}$ in approximation of the critical bands [6], and by quantizing the SPL values $\mathbf{L}$ with a step size equal to some value $\Delta$, which is put into relation to an appropriate psychoacoustic criterion.

The peripheral auditory system is usually modeled as a bank of overlapping bandwidth filters, the auditory filters, which possess an equivalent rectangular bandwidth (ERB). The scale that relates the center frequency of auditory filters to units of the ERB is the ERBrate scale. Using the ERB-rate function [8] we can define a relation between the frequency index $k$ and the frequency band index $z_{k}$, e.g., by

$$
z_{k}:=\left\lfloor 11.17 \ln \left|\frac{f_{s} / N k+0.312}{f_{s} / N k+14.68}\right|+43.0\right\rfloor,
$$

where $f_{s}$ is the sampling frequency in $\mathrm{kHz}$. The number of unique band indices $Z=\left|\left\{z_{k}\right\}\right|$ determines the size of the more compact 
energy matrix $\hat{\mathbf{E}}_{d \times Z}$,

$$
\hat{e}_{i, z}=\frac{1}{u(z)-l(z)+1} \sum_{k=l(z)}^{u(z)} E_{i}(m, k),
$$

with its $(i, z)^{\text {th }}$ entry calculated as the mean energy value between $l(z)=\inf \left\{k: z_{k}=z\right\}$ and $u(z)=\sup \left\{k: z_{k}=z\right\}$.

Acting on the assumption that the minimum just-noticeable difference level (JNDL) and so the maximum allowed quantization error is roughly $1 \mathrm{~dB}[6]$, the quantization step size $\Delta$ is set to $2 \mathrm{~dB}$, and the irrelevancy reduced SPL matrix $\mathbf{L}_{d \times Z}^{\Delta}$ is obtained from the following uniform quantizer:

$$
l_{i, z}^{\Delta}=\left\lfloor 5 \log _{10} \hat{e}_{i, z}\right\rceil .
$$

\subsection{Cluster-based iterative beamforming}

On the grounds that one interference signal can be suppressed by means of constraint beamforming based on the used data model, time-frequency points in the mixture spectrum are grouped together into disjoint clusters, which are distinguished by the number $M$ and the azimuth of the sound sources that prevail in these spectral areas:

$$
\mathcal{C}: M(m, k) \times \boldsymbol{\theta}_{M}(m, k) \mapsto c .
$$

The latter are derived from the known energy spectra, by finding the $M$ sources $(M \leqslant d)$, for which the signal-to-interference ratio (SIR),

$$
\operatorname{SIR}_{i}(m, k)=E_{i}(m, k) / \sum_{j=1, j \neq i}^{d} E_{j}(m, k),
$$

is greater than, e. g., $40 \mathrm{~dB}$ below the peak SIR in that point. Lowresolution energy spectra are extrapolated to STFT resolution by setting $E_{i}\left(m,\left\{k: z_{k}=z\right\}\right)=\hat{E}_{i}(m, z)$. For the purpose of algorithmic simplicity, points with $M>2$ are analyzed in respect of the two most predominant ones. The primary sound source is the source-of-interest (SOI), and an optional secondary source is the jammer. Points without any significant source contribution form the "zero cluster". The number of clusters is given by the number of possible source combinations, which is $C=\left(\begin{array}{c}d+1 \\ 2\end{array}\right)$. A similar clustering strategy, in which an ill-posed problem is reformulated as multiple well-posed problems, is pursued in [9], e. g.

Once the clusters $\left\{C_{0}, C_{1}, C_{2}\right\}$ are defined, source components in each 1-source cluster $c \in \mathrm{C}_{1}$ are estimated using the minimum variance distortionless response (MVDR) filter [10]. The optimum weight vector for a 2-source cluster $c \in \mathrm{C}_{2}$ is derived analogously, using the method of Lagrange multipliers:

$$
\mathbf{w}_{i, c}=\mathbf{C}_{X X, c}^{-1} \mathbf{A}_{c}\left(\mathbf{A}_{c}^{\mathrm{T}} \mathbf{C}_{X X, c}^{-1} \mathbf{A}_{c}\right)^{-1} \mathbf{g},
$$

where $\mathbf{C}_{X X, c}=\mathrm{E}\left(\mathbf{X}_{c}^{\emptyset} \mathbf{X}_{c}^{\emptyset \mathrm{H}}\right)$ is the $2 \times 2$ cluster covariance matrix, $\mathrm{E}$ is the expectation operator, $\mathbf{X}_{c}^{\emptyset}$ is the cluster data matrix, $\mathbf{A}_{c}=$ $\left[\begin{array}{ll}\mathbf{a}_{i} & \mathbf{a}_{j}\end{array}\right]$ is a submatrix of the mixing matrix $\mathbf{A}$, and $\mathbf{g}=\left[\begin{array}{ll}1 & 0\end{array}\right]^{\mathrm{T}}$ is the gain vector enforcing that the SOI at $\theta_{i}$ is preserved and the jammer at $\theta_{j}$ is suppressed (see Fig. 3). When the estimated cluster spectra $\left\{\mathbf{y}_{i, c}=\mathbf{w}_{i, c}^{\mathrm{T}} \mathbf{X}_{c}^{\emptyset}\right\}$ are pieced together, they form the complete SOI signal spectrum $\mathbf{y}_{\mathrm{SOI}}=\bigsqcup_{c=1}^{C} \mathbf{y}_{\mathrm{SOI}, c}$.

Both the clustering and the beamforming is executed once per iteration, as indicated by the superscript $n$ in Fig. 4. The purpose of

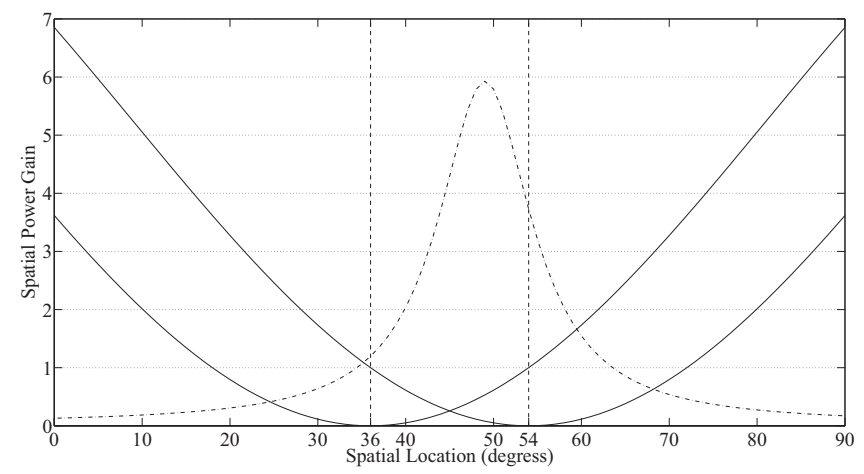

Figure 3: Exemplary beampatterns and power spectrum for a 2source cluster $\left(\theta_{i}=54^{\circ}, \theta_{j}=36^{\circ}\right)$.

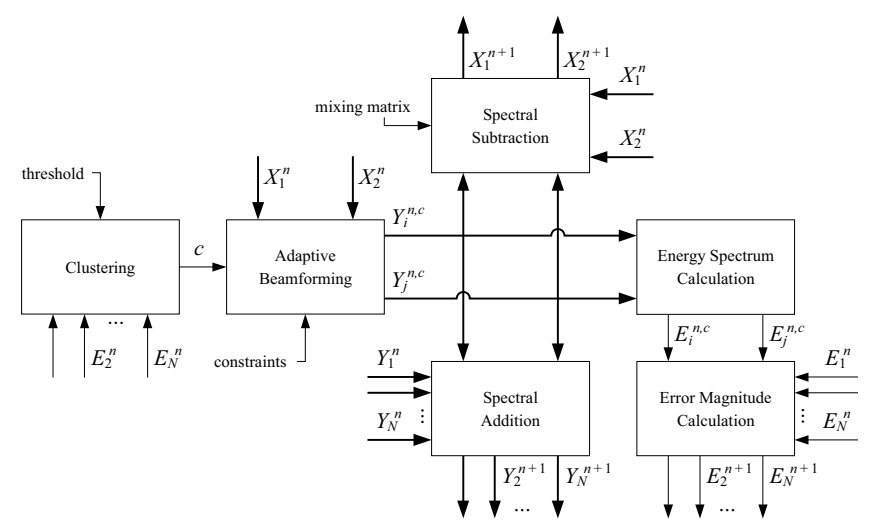

Figure 4: Block diagram of cluster-based iterative beamforming.

iterative filtering is to regain signal components from TF points with more than two active sources, by subtracting the estimated source signal spectra of the two most predominant sound sources from the mixture spectrum in each cycle, and to proceed until the impact of the least predominant source was taken into account. Accordingly, the affected energy spectra are readjusted in each cycle as well, for the the reason that the latter guide the cluster analysis:

$$
E_{i}^{n+1}(m, k)=\left.\left|E_{i}^{n}(m, k)-\right| Y_{i}^{n}(m, k)\right|^{2} \mid .
$$

\subsection{Spectral hole filling}

The term "spectral hole filling" found in Fig. 2 refers to the postbeamforming filter applied before the energy spectra are readjusted. As the name alludes, spectral holes are points $\{(m, k)\}$ in the timefrequency space, in which the modulus of the estimated spectrum $\left|Y_{i}(m, k)\right|$ is close to zero, whereas the original envelope $E_{i}(m, k)$ suggests different. They are due to approximation and estimation errors, as extrapolated envelopes yield a coarse approximation of the true energy spectra, which leads to inaccurate clustering on the one hand, and the fact that only one jammer is perfectly canceled, while others (if existent) are multiplied by a gain value other than zero, evokes faulty spectrum component estimates on the other. A naive approach is, e. g., to fill spectral holes with complex random values $V$ that have a standard normal amplitude, $|V| \sim \mathcal{N}(0,1)$, and a uniform phase, $\arg V \sim \mathcal{U}(0,2 \pi)$, which in fact means to replace missing signal components by (colored) narrowband noise. 


\begin{tabular}{lcccccc}
\hline & \multicolumn{2}{c}{ SNR $(\mathrm{dB})$} & \multicolumn{2}{c}{ SNR $^{\mathrm{t}}(\mathrm{dB})$} & \multicolumn{2}{c}{ PSM $(\%)$} \\
\cline { 2 - 7 } & ISSA & USSR & ISSA & USSR & ISSA & USSR \\
\hline Accapela & $\mathbf{1 2 . 2}$ & $8.05(8.99)$ & -9.67 & $\mathbf{1 . 5 4}(2.20)$ & 91 & $\mathbf{9 8}(98)$ \\
Bass & 10.5 & $\mathbf{1 0 . 6}(9.71)$ & -1.86 & $\mathbf{7 . 5 7}(7.68)$ & 80 & $\mathbf{9 6}(97)$ \\
Beat & $\mathbf{1 3 . 6}$ & $8.99(8.82)$ & -1.75 & $\mathbf{5 . 7 0}(6.45)$ & 79 & $\mathbf{9 1}(92)$ \\
Goblins & 6.97 & $\mathbf{7 . 3 2}(8.27)$ & 7.54 & $\mathbf{8 . 4 0}(9.85)$ & 73 & $\mathbf{9 6}(96)$ \\
Guitar & 7.42 & $\mathbf{8 . 2 4}(9.53)$ & -4.09 & $\mathbf{5 . 6 1}(6.67)$ & 90 & $\mathbf{9 8}(99)$ \\
Keys + CS & $\mathbf{2 . 1 1}$ & $1.94(2.77)$ & -0.56 & $\mathbf{3 . 6 0}(4.53)$ & 52 & $\mathbf{9 4}(95)$ \\
\hline
\end{tabular}

Table 1: SNR and PSM values for an excerpt from "Armistice" by Phoenix. Best values are boldfaced. Full-resolution USSR values are in brackets.

\section{SIMULATION RESULTS}

The proposed “USSR" algorithm was programmed in MATLAB ${ }^{\circledR}$ and compared with "ISSA" [4] under the following conditions: The STFT was implemented as a 2048-point discrete Fourier transform (DFT) with a Kaiser-Bessel derived (KBD) window of the same length and an $\alpha$ of 20. The overlap between consecutive data blocks was $50 \%$. The sampling rate was set to $44.1 \mathrm{kHz}$. An excerpt of $38 \mathrm{~s}$ from a multitrack recording composed of 6 simultaneously emitting sources was considered. Single tracks were associated to the pan angles $48,54,45,18,36$, and 68 , all given in degrees. USSR was run in a low-resolution mode with 72 frequency bands and a fullresolution mode for reference. Both ISSA and USSR processed the entire Nyquist spectrum. The per-channel data rate totaled up to $108 \mathrm{kbps}$ for ISSA and $54.5 \mathrm{kbps}$ in case of low-resolution USSR, using 16-bit pulse-code modulation (PCM) with a dynamic range of $96 \mathrm{~dB}$. The data rate for full-resolution USSR is in the region of linear PCM and has no practical use.

The systems under test were first compared in terms of the signal-to-noise ratio (SNR) between the original signal $\mathbf{s}_{i}$ and the estimation error $\left(\hat{\mathbf{s}}_{i}-\mathbf{s}_{i}\right)$ as per

$$
\mathrm{SNR}_{i}=\frac{\left\|\mathbf{s}_{i}\right\|_{2}^{2}}{\left\|\hat{\mathbf{s}}_{i}-\mathbf{s}_{i}\right\|_{2}^{2}},
$$

by considering the global SNR value for the entire signal, and by calculating the mean of local SNR values in 10-ms long intervals. The latter is labeled $\mathrm{SNR}^{\mathrm{t}}$, and the picked up results are summarized in Tab. 1. Even though the global SNR suggests a good performance for ISSA, all of its SNR ${ }^{t}$ values, with the exception of the "Goblins" track, fall below the $0 \mathrm{~dB}$ mark. The $\mathrm{SNR}^{\mathrm{t}}$ measure can hence be seen as a first indicator of missing sound components. Tab. 1 shows furthermore that with regard to $\mathrm{SNR}^{\mathrm{t}}$, USSR performs clearly better with full as well as low frequency resolution. PEMO-Q [11] was used thereafter to predict perceived quality degradation between the original signals and their replica, which is expressed in terms of the perceptual similarity measure (PSM). As is evident from Tab. 1, signals recovered with the USSR technique bear a higher perceptual resemblance to the original. Also remarkable is that the low-bitrate version of USSR shows as few as $1 \%$ deficit compared to the fullresolution version at a bitrate gain of more than $500 \mathrm{kbps}$. Informal listening tests further suggest that the quality impairment due to reconstruction errors is less annoying than the arid effect observed in [4]. ${ }^{1}$

\footnotetext{
1http://www.labri.fr/ gorlow/waspaa11/
}

\section{CONCLUSIONS}

By the present work we have presented a novel approach, which solves the ill-posed source separation problem, without making any assumptions about the number of locally predominant sound sources. The method makes use of psychological phenomena of the auditory system in order to derive a compact set of parameters that help reconstruct a perceptually close copy of original tracks based on the mixture signal, while eliminating the arid effect. By means of an objective measure it was demonstrated that a higher similarity to the original signals is achieved in comparison to a similar state-ofthe-art method at half the data rate. A shortcoming of the method is that losses in harmonic structure cannot yet be compensated for by the suggested post-beamforming filter. Formal listening tests have yet to be carried out to assess the audio quality more objectively.

\section{ACKNOWLEDGMENTS}

This research was partly supported by the French ANR DReaM project (ANR-09-CORD-006).

\section{REFERENCES}

[1] P. Comon and C. Jutten, Handbook of Blind Source Separation: Independent Component Analysis and Applications, 1st ed. Academic Press, 2010.

[2] S. Marchand, B. Mansencal, and L. Girin, "Interactive music with active audio CDs," in Proc. CMMR, 2010, pp. 73-74.

[3] S. Makino, T.-W. Lee, and H. Sawada, Blind Speech Separation. Springer, 2007.

[4] M. Parvaix and L. Girin, "Informed source separation of linear instantaneous under-determined audio mixtures by source index embedding," IEEE Trans. Audio Speech Lang. Process., vol. 19, pp. 1721-1733, Aug. 2011.

[5] A. Liutkus, R. Badeau, and G. Richard, "Informed source separation using latent components," in Proc. LVA/ICA, 2010, pp. 498-505.

[6] H. Fastl and E. Zwicker, Psychoacoustics: Facts and Models, 3rd ed. Springer, 2007.

[7] S. Rickard and O. Y1lmaz, "On the approximate W-disjoint orthogonality of speech," in Proc. IEEE ICASSP, 2002, pp. 529-532.

[8] B. C. J. Moore and B. R. Glasberg, "Suggested formulae for calculating auditory-filter bandwidths and excitation patterns," J. Acoust. Soc. Am., vol. 74, pp. 750-753, Sept. 1983.

[9] M. I. Mandel, R. J. Weiss, and D. P. W. Ellis, "Model-based expectation-maximization source separation and localization," IEEE Trans. Audio Speech Lang. Process., vol. 18, pp. 382394, Feb. 2010.

[10] J. Capon, "High-resolution frequency-wavenumber spectrum analysis," in Proc. IEEE, 1969, pp. 1408-1418.

[11] R. Huber and B. Kollmeier, "PEMO-Q - a new method for objective audio quality assessment using a model of auditory perception," IEEE Trans. Audio Speech Lang. Process., vol. 14, pp. 1902-1911, Nov. 2006. 